\section{Особенности влияния основных факторов на глубину проникновения пропиточной жидкости}

\author{
В. А. Новиков \\ Петрозаводский государственный университет
}

\begin{abstract}
АННОТАЦИЯ
В статье представлены результаты исследований прессового и фронтального способов пропитки. Показаны степень и характер влияния основных значимых факторов. В качестве инструмента исследований используется математическая модель процесса пропитки в упругомеханическом поле, полученная ранее Исследования имеют не только теоретическое значение, но и представляют практический интерес.
\end{abstract}

Ключевые слова: защитная обработка, пропитка древесины, математическая модель.

\section{SUMMARY}

The process of impregnation of timber is investigated. The mathematical model of process of impregnation was earlier built. The outcomes of research of the major factors are submitted. The analysis of influencing of major factors on depth of penetration of treating liquid is carried out. The research is not only of theoretical significance but has some practical value.

Keywords: protective processing, impregnation of timber, mathematical model.

\section{ВВЕДЕНИЕ}

Математическая модель пропитки древесины с использованием упругих элементов с системой тупиковых отверстий позволяет рассчитывать глубину проникновения пропитывающей жидкости в зависимости от различных факторов $[1,2]$. Изменение того либо другого фактора в разной степени влияет на определяемые параметры пропитки. Исследование степени влияния каждого из них является важным звеном в вопросе изучения процессов движения жидкости в древесине в результате избыточного давления.

\section{ЗАВИСИМОСТЬ ГЛУБИНЫ ПРОПИТКИ ОТ ОСНОВНЫХ ПОКАЗАТЕЛЕЙ}

На глубину проникновения пропитывающей жидкости в результате избыточного давления определяющим образом влияют следующие факторы $[1,2,3]$ : - коэффициент проницаемости древесины $\boldsymbol{C}$, который, в свою очередь, зависит, например, от породы, температуры образца, места выпила, качества древесины и т.д.;

\footnotetext{
' Автор - дочент кафедры промыиленного транспорта и геодезии
}

(C) В. А. Новиков, 2001
- значение величины максимального давления $\boldsymbol{P}_{\text {мax }}$, прикладываемого к упругому элементу;

- значение коэффициента воздухоемкости древесины $\boldsymbol{B}_{W}$;

- значение коэффициента плотности пропитывающей жидкости $\rho$;

- значение коэффициента абсолютной вязкости пропитывающей жидкости $v$;

- ускорение силы тяжести $\boldsymbol{g}$;

- характеристики упругого элемента $\boldsymbol{h}, \boldsymbol{\Delta h}, \boldsymbol{R}$;

- значение коэффициента $\boldsymbol{\Pi}_{\boldsymbol{y}}$, учитывающего пористость упругого элемента;

- значение модуля упругости упругого элемента $\boldsymbol{E}_{\boldsymbol{Y}}$;

- значение коэффициента, учитывающего характер нагружения упругого элемента, скорость изменения величины деформации или скорость протягивания через вальцы $\boldsymbol{b}, \boldsymbol{v}$;

- значение коэффициента предварительного нагружения упругого элемента $\boldsymbol{A}$;

- значение величины предварительно пропитанного слоя жидкости в древесине $\Delta \delta_{\text {l. }}$.

Изменение каждого из них в разной степени влияет на глубину проникновения пропиточной жидкости. Кроме того, для пропитки древесины представленные выше параметры и коэффициенты имеют реальные границы, в пределах которых их необходимо рассматривать. Как показывают экспериментальные исследования [2, 3], определяющее значение на выходные параметры пропитки оказывают: коэффициент проницаемости $\boldsymbol{C}$; модуль упругости упругого элемента $\boldsymbol{E}_{\boldsymbol{Y}}$; коэффициент воздухоемкости древесины $\boldsymbol{B}_{W}$; коэффициент пористости упругого элемента $\boldsymbol{\Pi}_{\boldsymbol{y}} ;$ величина деформации упругого элемента $\Delta \boldsymbol{h}$, время нагружения $\boldsymbol{t}$, время выдержки упругого элемента под максимальной нагрузкой $\boldsymbol{t}_{\boldsymbol{l}}$.

С использованием математической модели $[1,2]$ были получены зависимости влияния наиболее значимых из перечисленных коэффициентов на глубину проникновения пропитывающей жидкости.

На рисунках 1, 2 в графическом виде показана степень влияния времени нагружения $\boldsymbol{t}$ и времени выдержки максимального давления $\boldsymbol{t}_{\text {/ }}$ для различных образцов березы в тангентальном направлении пропитки на глубину проникновения пропиточной жидкости $\Delta \delta$. Постоянные значимые для процесса факторы в этой серии расчётов были приняты со следующими значениями: $\boldsymbol{B}_{W}=0,66 ; \rho=10^{3} \mathrm{\kappa r} / \mathrm{M}^{3} ; \quad \Delta \delta_{\theta}=$ $0,0001 \mathrm{м} ; \boldsymbol{\Pi}_{\boldsymbol{V}}=0,02 ; \boldsymbol{h}=0,06 \mathrm{м} ; \boldsymbol{g}=9,81 \mathrm{м} /$ сек.

Анализируя графики на рисунках 1 и 2 , можно сделать вывод о степени влияния важнейшего фактора $\boldsymbol{P}_{\text {мax }}$ - величины максимального давления, прикладываемого к упругому элементу. На рисунке 1 представлен график зависимости глубины пропитки при минимальном значении $\boldsymbol{P}_{\text {мax }}=1 \mathrm{MПа}$, а на рисунке 2 при максимальном значении $\boldsymbol{P}_{\boldsymbol{s u x}}=5$ МПа при различных начальных параметрах коэффициента проницаемости. 


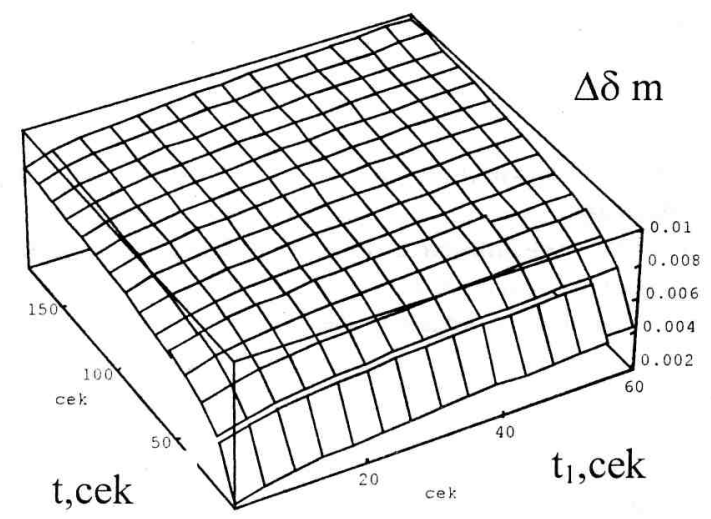

Рис. 1. Зависимость глубины пропитки $\Delta \delta$ от времени нагружения упругого элемента $t$ до максимального значения и времени выдержки $\boldsymbol{t}_{t}$. Способ пропитки прессовый. $\boldsymbol{P}_{\text {мax }}$ - 1 МПа. Береза, ядро, тангентальное направление пропитки.

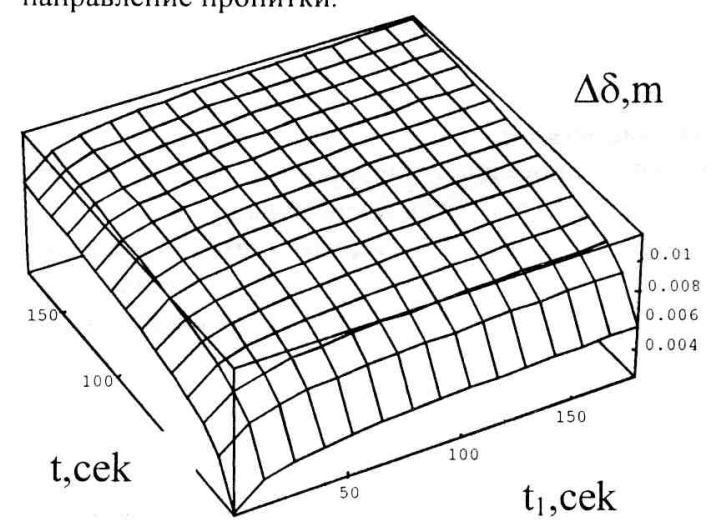

Рис. 2. Зависимость глубины пропитки $\Delta \delta$ от времени нагружения упругого элемента $t$ до максимального значения и времени выдержки $\boldsymbol{t}_{1}$. Способ пропитки прессовый. $\boldsymbol{P}_{\text {мax }}-5$ МПа. Береза, ядро, тангентальное направление пропитки.

Отметим, что максимальная интенсивность проникновения пропиточной жидкости наблюдается на начальной стадии процесса. При увеличении времени пропитки интенсивность проникновения жидкости со временем снижается. Это объясняется, в первую очередь, особенностями процесса пропитки с использованием упругих элементов. По мере выдавливания пропиточной жидкости из глухих отверстий изменяется, в сторону уменьшения, величина избыточного давления на границе пропитывающей жидкости и древесины. И, во-вторых, происходит изменение значения коэффициента проницаемости древесины в силу того, что пропитывающая жидкость заполняет поверхностный слой древесины.

На рисунках 3 и 4 представлены зависимости глубины проникновения пропиточной жидкости от значения коэффициента проницаемости древесины $\boldsymbol{C}$ и значения коэффициента воздухоемкости $\boldsymbol{B}_{W}$ при постоянных значениях остальных значимых для процесса факторов.

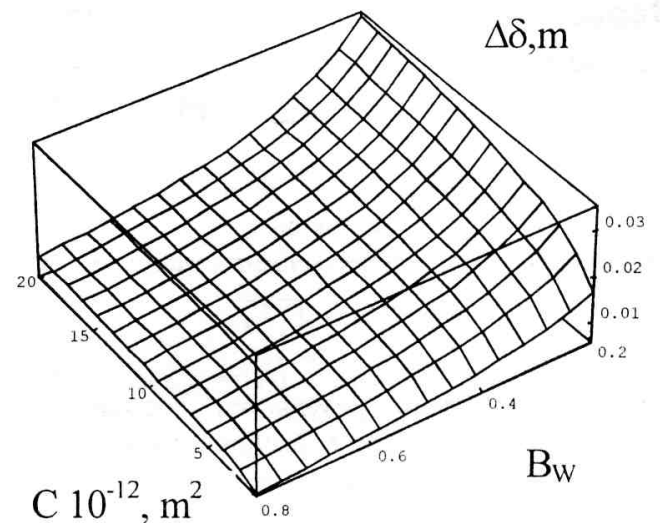

Рис. 3. Зависимость глубины пропитки $\Delta \delta$ от коэффициента проницаемости $\boldsymbol{C}$ и коэффициента воздухоемкости $\boldsymbol{B}_{W}$ при минимальных значениях $\boldsymbol{C}$ и $\boldsymbol{B}_{\boldsymbol{W}}$.

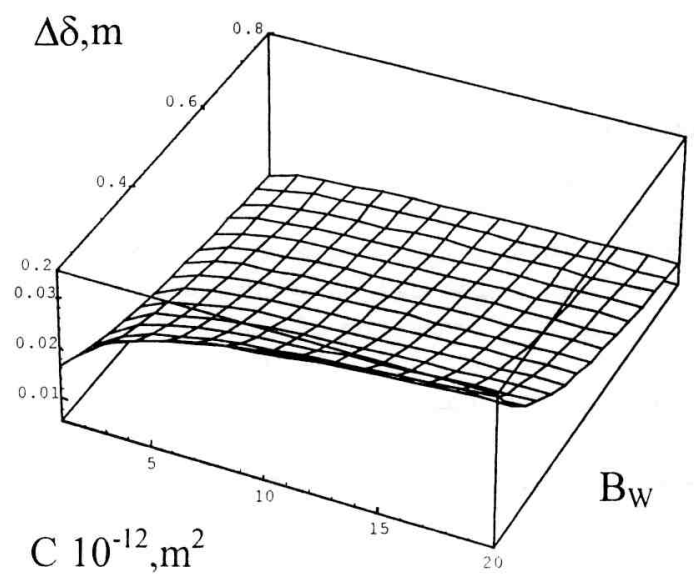

Рис. 4. Зависимость глубины пропитки $\Delta \delta$ от коэффициента проницаемости $C$ и коэффициента воздухоемкости $\boldsymbol{B}_{W}$ при максимальных значениях $\boldsymbol{C}$ и $\boldsymbol{B}_{\boldsymbol{W}}$.

Принималось: $\boldsymbol{C}=1 \times 10^{-12}-20 \times 10^{-12} \mathrm{M}^{2} ; \boldsymbol{B}_{\boldsymbol{W}}=0.2-0.8$; $\boldsymbol{P}_{\text {. } a x}=5 \mathrm{MПа} ; \quad \rho=10^{3} \mathrm{\kappa r} / \mathrm{M}^{3} ; \quad \Delta \delta_{0}=0,0001 \mathrm{M} ; \quad \Pi_{\boldsymbol{V}}=$

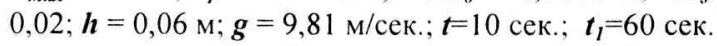

Рассмотрение этих величин во взаимосвязи обусловлено тем, что коэффициент проницаемости древесины и коэффициент воздухоемкости зависят главным образом от свойств пропитываемого образца, качества обработки поверхности и в конечном итоге влияют друг на друга. Отметим, что значения этих величин изменяются в течение всего процесса.

Характер же изменения глубины пропитки остается постоянным при различных значениях коэффициента проницаемости и коэффициента воздухоемкости, это замечание наглядно проиллюстрировано на представленных рисунках.

Покажем для сравнения изменение глубины проникновения пропиточной жидкости в поверхностный слой древесины в результате многократного нагружения упругого элемента при следующих закреплен- 
ных параметрах: $\boldsymbol{C}=0,9 \times 10^{-12} \mathrm{~m}^{2} ; \quad \boldsymbol{B}_{W}=0,66 ; \quad \boldsymbol{t}=10$ сек; $\boldsymbol{t}_{\boldsymbol{l}}=60$ сек; $\rho=10^{3} \mathrm{\kappa} / \mathrm{M}^{3} ; \Delta \delta_{l}=0,0001 \mathrm{м}$; $\boldsymbol{\Pi}_{V}=0,02 ; \boldsymbol{h}=0,06 \mathrm{м} ; \boldsymbol{g}=9,81 \mathrm{м} /$ сек.; $\boldsymbol{t}=10$ сек.; $\boldsymbol{t}_{\boldsymbol{l}}=60$ сек. (рис. 5.).

Представленная схема процесса может быть применена в том случае, если наблюдается необходимость в более глубокой пропитке. Объема жидкости, находящегося в глухих отверстиях, на один цикл может быть недостаточно, и тогда для наполнения отверстий необходимо снять нагрузку с упругого элемента. Так как упругий элемент находится в ванне с пропиточным составом, он опять заполнит тупиковые отверстия, восстановив первоначальный объем. Подобный цикл можно повторять многократно, учитывая при этом, что с каждым новым циклом количество жидкости, вошедшей в древесину, будет уменьшаться. Это объясняется тем, что значение коэффициента проницаемости $\boldsymbol{C}$ обрабатываемого образца снижается.

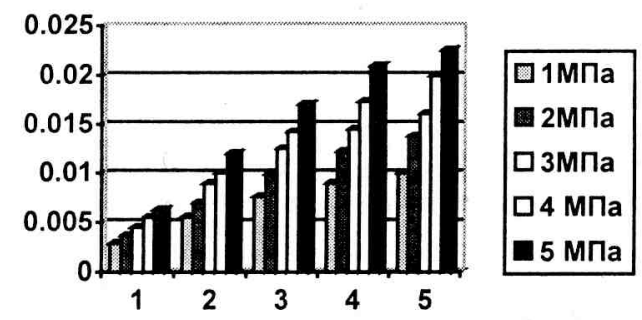

Рис. 5. Зависимость глубины пропитки $\Delta \delta$ для прессового способа от величины максимального давления $\boldsymbol{P}_{\text {мах }}$, прикладываемого к упругому элементу, и количества нагружений $N$.

Для фронтального способа пропитки, при котором максимальное значение давления пропитывающей жидкости в тупиковых отверстиях достигается посредством деформации упругих покрытий вальцов, определяюшим фактором является степень максимальной деформации упругого покрытия вальца. Величина деформации непосредственно влияет на величину давления пропитывающей жидкости на границе контакта пропитывающей жидкости и древесины. Скорость протягивания образца прямым образом связана со скоростью изменения величины деформации упругого покрытия вальца. Диаметр вальцов главным образом влияет на величину площади контакта упругой поверхности вальца с поверхностью древесины и тем самым, учитывая дополнительно угловую скорость вальцов, на время контакта с единичной поверхностью.

Очевидно, что чем выше значения модуля упругости, площади контакта и меньше линейная скорость протягивания образцов, тем, при равных прочих постоянных параметрах, будет интенсивность пропитки выше. В этом случае и значение глубины пропитки будет наибольшим.
Покажем характер влияния вышеперечисленных факторов на выходной параметр процесса пропитки фронтальным способом.

На рисунках 6 и 7 в графическом виде представлена зависимость глубины проникновения пропиточной жидкости от диаметра контактирующего вальца и скорости протягивания заготовок.

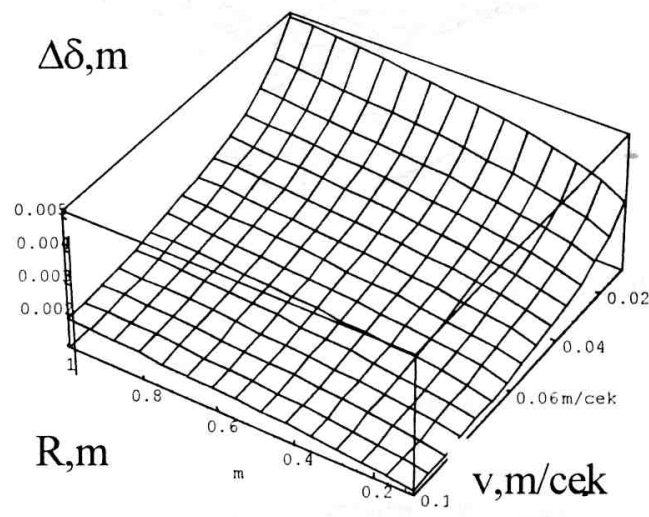

Рис. 6. Зависимость глубины пропитки $\Delta \delta$ для фронтального способа от радиуса контактирующего вальца $\boldsymbol{R}$, а также скорости протягивания образца $v$ при минимальном значении $\boldsymbol{R}$ и максимальном значении $\boldsymbol{v}$.

Зона контакта упругого покрытия вальца и поверхности древесины определяется по формуле:

$$
S=l d \quad\left(\mathrm{M}^{2}\right),
$$

где $\boldsymbol{l}$ - длина зоны контакта;

$$
\begin{aligned}
l=2 \sqrt{R^{2}-\left(R-\Delta h_{\max }\right)^{2}} & \text { (м), } \\
& \boldsymbol{d} \text { - ширина протягиваемого образца; } \\
& \boldsymbol{R} \text { - радиус контактирующего с обрабаты- }
\end{aligned}
$$

ваемой поверхностью вальца;

$$
\Delta \boldsymbol{h} \text { - величина деформации вальца. }
$$

Время контакта определяется из соотношения:

$$
t=\frac{l}{v} \text { (сек.), }
$$

где $v$ - скорость протягивания.

Скорость изменения величины деформации:

$b=\frac{\Delta h_{\max }}{t} \quad$ (м/сек.),

где $\boldsymbol{t}$ - время достижения максимальной деформации упругого элемента на единичной поверхности с начала контакта. 


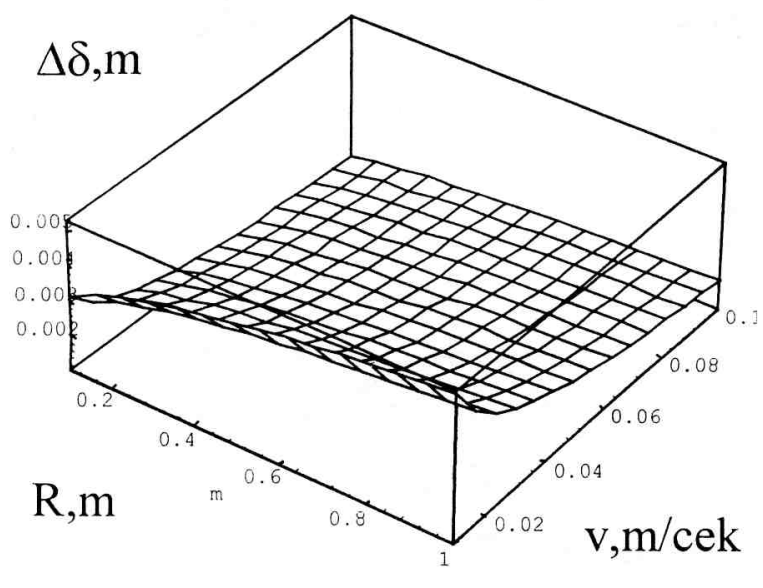

Рис. 7. Зависимость глубины пропитки $\Delta \delta$ для фронтального способа от радиуса контактирующего вальца $\boldsymbol{R}$, а также скорости протягивания образца $v$ при максимальном значении $\boldsymbol{R}$ и минимальном значении $v$.

При исследовании фронтального способа принимались следуюшие значения постоянных параметров: $\boldsymbol{C}=0,9 \times 10^{-12} \mathrm{~m}^{2} ; \boldsymbol{B}_{W}=0,66 ; \rho=10^{3} \mathrm{\kappa} / \mathrm{M}^{3} ; \boldsymbol{P}_{\text {мax }}=1 \mathrm{MПа;}$ $\boldsymbol{E}=40 \times 10^{-12}$ МПа; $\Delta \delta_{l}=0,0001 \mathrm{м} ; \boldsymbol{\Pi}_{\boldsymbol{y}}=0,02$; $\boldsymbol{h}=0,06 \mathrm{M} ; \boldsymbol{R}=0,1-1 \mathrm{M} ; \boldsymbol{v}=0,01-0,1 \mathrm{M} /$ сек.

Варьируя параметрами диаметра контактирующего вальца и скоростью протягивания образцов, можно добиться требуемой глубины проникновения пропитывающей жидкости исходя из нужд реального производства.

Аналогичным образом можно контролировать глубину пропитки, если уменьшать либо увеличивать количество контактирующих с обрабатываемой поверхностью пар вальцов. Одновременно возможно изменение величины деформации упругого покрытия вальца. Вместе с этим происходит изменение значения давления пропитывающей жидкости в глухих отверстиях упругого покрытия.

На рисунке 8 представлены зависимости глубины пропитки фронтальным способом при изменении количества пар вальцов с 1 до 10 и одновременном изменении величины максимального давления от 1 МПа до 5 МПа. Скорость протягивания образца принята $v=0,1 \mathrm{~m} /$ сек.
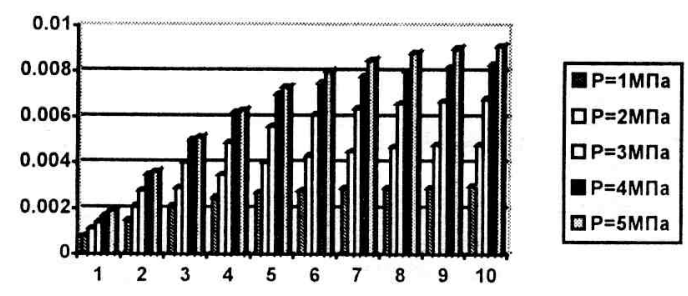

Рис. 8. Зависимость глубины пропитки $\Delta \delta$ для фронтального способа от величины максимального давле- ния $\boldsymbol{P}_{\boldsymbol{M a x}}$, прикладываемого к упругому элементу, и количества пар контактирующих вальцов.

Аналитические зависимости позволяют рассчитывать параметры пропитки для каждой последующей пары с учетом того, что в них учитывается предварительно пропитанный слой жидкости.

Из проведенных аналитических расчетов прессового и фронтального способов можно сделать вывод о том, что максимальное значение глубины проникновения пропитывающей жидкости в поверхность древесины наблюдается в начальный период времени. Это подтверждается и исследованиями других авторов [3]. На практике этот эффект усиливается тем, что в начальный момент времени силы капиллярного давления имеют максимальное значение

Рассмотренная методика определения наиболее значимых коэффициентов в аналитических зависимостях глубины пропитки и оценка характера влияния основных факторов на выходные параметры пропитки помогают полнее представить механизм проникновения жидкости в древесину в результате избыточного давления, позволяют определять оптимальные параметры пропиточной установки, использующей принцип пропитки древесины в упругомеханическом поле в цепочке конкретного технологического производства.

\section{ЛИТЕРАТУРА}

1. Новиков В. А. Определение выходных параметров при защитной обработке изделий из древесины в упругомеханическом поле // Труды лесоинженерного факультета ПетрГУ. Вып. 1. Петрозаводск: Изд-во ПетрГУ, 1997. С. 25-27.

2. Новиков В. А. Определение параметров пропитки изделий из древесины в упругомеханическом поле. М., 1998. Деп. в ВИНИТИ. № 2287-В98,.

3. Оснач Н. А. Проницаемость и проводимость древесины. М.: Лесная промышленность, 1964. $128 \mathrm{c}$. 